\title{
Understanding the Moral Attitudes Scale in International Surveys: an Exploratory Study
}

\author{
Alisa Remizova ${ }^{1}$ \\ Email: aremizova@hse.ru (corresponding author) \\ Maksim Rudnev ${ }^{1,2}$ \\ Email: mrudnev@hse.ru,
}

ORCID: 0000-0002-2714-3840

1 National Research University Higher School of Economics, Moscow, Russia

2 Instituto Universitário de Lisboa (ISCTE-IUL), CIS-IUL, Lisbon, Portugal

Acknowledgements

This article is an output of a research project implemented as part of the Basic Research Program at the National Research University Higher School of Economics (HSE University).

The authors are grateful to Vladimir Magun for his useful comments on the earlier versions of the manuscript. 


\begin{abstract}
Several large international surveys, including the World Values Survey and the European Values Study, have been using the moral attitudes scale (MAS) to measure individual and country differences for decades. However, the validity of the instrument has been barely assessed. The current study addressed the concurrent and content validity of four popular MAS items (justifiability of homosexuality, suicide, prostitution, and euthanasia). A sample of 493 Russians completed both MAS and the four validated multi-item scales. Results demonstrated that, except for the homosexuality item, the MAS items had low concurrent validity, explaining less than $50 \%$ of the variance of the corresponding multi-item scales. The MAS items underestimated the justifiability of homosexuality, prostitution, and suicide, and overestimated the justifiability of euthanasia. The MAS homosexuality item appeared to be a precise measure of attitude towards male (but not female) homosexuality, responses to the prostitution and suicide items overlooked the positive arguments, and the euthanasia item tapped more into attitudes towards euthanasia of a dying person. The four items showed strong dependence on the overall justifiability. We conclude that separate MAS items should be used with caution, given the detected content bias and the items' strong link to non-specific overall justifiability.
\end{abstract}

Keywords: European Values Study, Moral Attitudes Scale, justifiability, concurrent validity 


\section{INTRODUCTION}

The World Values Survey (WVS, 2018) and the European Values Study (EVS, 2018) are large repeated international surveys. They have been collecting data using national representative samples since 1981, both in European countries and globally, exploring people's attitudes across over one hundred nations. The data collected by these surveys have been used in a large number of individual studies across several disciplines. Nonetheless, the validity and reliability of many of the measures used in these surveys are rarely investigated. In particular, the Moral Attitudes Scale (MAS), a part of the EVS and the WVS, has unknown reliability and validity. Nevertheless, various MAS items are often used to describe and explain moral attitudes, focusing on the justifiability of homosexuality (e.g. Adamzcyk and Pitt 2009; Štulhofer and Rimac 2009), prostitution (e.g. Cao, Lu, and Mei 2017), suicide (Stack and Kposowa 2016), euthanasia (e.g. Rudnev and Savelkaeva 2018), tax evasion (Alm and Torgler 2006), divorce (Kalmijn 2009), drug use (Cao and Zhao 2012), etc. Additionally, the MAS items are used to create composite scores of overall justifiability (e.g. Vauclair and Fischer 2011; Chen 2014; Lottes and Alkula 2011) or included in broader indices - for example, the MAS homosexuality item has been listed among the prominent measures of self-expression (Inglehart and Baker 2000) and emancipative values (Welzel 2013). Unfortunately, both the "battery" format of the MAS and the use of single items as measures of standalone concepts may have incurred a significant yet unnoticed bias.

Validity of measurement is a fundamental requirement, and the lack of it may potentially undermine the conclusions of studies that use MAS items. The purpose of the current study is to assess the extent and content of the bias evoked by the battery format and single-item measurement. We focus on the investigation of concurrent and content validity of four MAS items - homosexuality, prostitution, suicide, and euthanasia — through their comparison with scales that have known reliability and validity.

The paper is structured as follows. The second part presents a brief review of the literature on the advantages and disadvantages of single-item and multi-item measurement, and of the problem of "satisficing". The third section describes the design of the empirical study, and its results are presented in the fourth part. The last section summarizes the results, gives recommendations, and suggests directions for future research. 


\section{BACKGROUND}

\section{The Moral Attitudes Scale}

The MAS instrument, originally labeled "Morally Debatable Behaviors Scale", was first developed in 1942 (Crissman 1942) and then adapted for use specifically in the EVS (Harding, Phillips, and Fogarty 1986). Currently, it is presented in a shorter form in WVS and in a longer form in EVS questionnaires. MAS is a list of generally unrelated (but sometimes and in some societies considered morally ambiguous) aspects of life, such as lying, homosexuality, tax evasion or euthanasia. The list of items is preceded by a general question of whether each of these aspects is justified or not, on the ten-point scale from "never justified" to "always justified". The number of items varies across the waves of surveys as well as across countries (for example, the homosexuality item was excluded from the WVS questionnaire in some Muslim countries). Table 1 lists the 20 items included in the $4^{\text {th }}$ wave of the EVS.

Such batteries of questions are a common data collection format (Siminski 2008) which has a number of known problems (Saris and Gallhofer 2014; Couper et al. 2013; Mavletova, Couper, and Lebedev 2018). The primary difficulty follows from the fact that the general information (introduction, the question itself, instructions, and response options) is not repeated before every single item. It can cause mixing of responses obtained by specific items with each other, as well as provoking less considered responses. As the items in MAS are thematically related, if only weakly, the problem of response mixing can be especially dangerous.

Beyond the battery format, the use of single items as measures of the specific latent constructs can lead to low content validity when only a part of the construct is actually assessed. Single-item measurement can also lead to low reliability because single items are more susceptible to situational influences and misinterpretation by respondents as compared to multiitem measurement. We explicate these two problems in the following sections. 
Table 1. Moral Attitudes Scale, European Values Study Wave 4 version (starred items are those tested in the current study)

Please tell me for each of the following whether you think it can always be justified, never be justified, or something in between, using this card.

\begin{tabular}{|c|c|c|c|c|c|c|c|c|c|c|}
\hline & Never & & & & & & & & & Always \\
\hline $\begin{array}{l}\text { Claiming state benefits which you are not } \\
\text { entitled to }\end{array}$ & 1 & 2 & 3 & 4 & 5 & 6 & 7 & 8 & 9 & 10 \\
\hline Cheating on tax if you have the chance & 1 & 2 & 3 & 4 & 5 & 6 & 7 & 8 & 9 & 10 \\
\hline $\begin{array}{l}\text { Taking and driving away a car belonging to } \\
\text { someone else (joyriding) }\end{array}$ & 1 & 2 & 3 & 4 & 5 & 6 & 7 & 8 & 9 & 10 \\
\hline Taking the drug marijuana or hashish & 1 & 2 & 3 & 4 & 5 & 6 & 7 & 8 & 9 & 10 \\
\hline Lying in your own interest & 1 & 2 & 3 & 4 & 5 & 6 & 7 & 8 & 9 & 10 \\
\hline Married men/women having an affair & 1 & 2 & 3 & 4 & 5 & 6 & 7 & 8 & 9 & 10 \\
\hline $\begin{array}{l}\text { Someone accepting a bribe in the course of } \\
\text { their duties }\end{array}$ & 1 & 2 & 3 & 4 & 5 & 6 & 7 & 8 & 9 & 10 \\
\hline *Homosexuality & 1 & 2 & 3 & 4 & 5 & 6 & 7 & 8 & 9 & 10 \\
\hline Abortion & 1 & 2 & 3 & 4 & 5 & 6 & 7 & 8 & 9 & 10 \\
\hline Divorce & 1 & 2 & 3 & 4 & 5 & 6 & 7 & 8 & 9 & 10 \\
\hline $\begin{array}{l}\text { *Euthanasia (terminating the life of the } \\
\text { incurably sick) }\end{array}$ & 1 & 2 & 3 & 4 & 5 & 6 & 7 & 8 & 9 & 10 \\
\hline *Suicide & 1 & 2 & 3 & 4 & 5 & 6 & 7 & 8 & 9 & 10 \\
\hline Paying cash for services to avoid taxes & 1 & 2 & 3 & 4 & 5 & 6 & 7 & 8 & 9 & 10 \\
\hline Having casual sex & 1 & 2 & 3 & 4 & 5 & 6 & 7 & 8 & 9 & 10 \\
\hline Avoiding a fare on public transport & 1 & 2 & 3 & 4 & 5 & 6 & 7 & 8 & 9 & 10 \\
\hline *Prostitution & 1 & 2 & 3 & 4 & 5 & 6 & 7 & 8 & 9 & 10 \\
\hline Scientific experiments on human embryos & 1 & 2 & 3 & 4 & 5 & 6 & 7 & 8 & 9 & 10 \\
\hline Genetic manipulation of food stuffs & 1 & 2 & 3 & 4 & 5 & 6 & 7 & 8 & 9 & 10 \\
\hline $\begin{array}{l}\text { Artificial insemination or in-vitro } \\
\text { fertilization }\end{array}$ & 1 & 2 & 3 & 4 & 5 & 6 & 7 & 8 & 9 & 10 \\
\hline Death penalty & 1 & 2 & 3 & 4 & 5 & 6 & 7 & 8 & 9 & 10 \\
\hline
\end{tabular}

\section{Single-item measurement of concepts and the problem of content validity}

The choice to measure a construct with a single item saves resources for data collection with large heterogeneous samples (Gorsuch and McPherson 1989). It may be sufficient where only a rough and general assessment is required, whereas obtaining detailed knowledge of the concept and identifying exact individual differences is not a primary goal (Fuchs and Diamantopoulos 2009; Churchill 1979).

At the same time, single-item measurement involves a number of risks. First, it requires a uniform understanding of an item by all respondents. This is not problematic if a researcher needs to measure some factual characteristic such as a respondent's age; that is, something perceived identically by the majority of respondents (Rossiter 2002). However, this is rarely the case. In the social sciences in general, as well as when using MAS items, researchers study complex latent constructs which manifest themselves in multiple forms (Alwin and Krosnick 
1991). Differences in interpretation of an item across respondents cause a large amount of noise, which by its nature undermines reliability of measurement (De Leeuw, Hox, and Dillman 2012; Steyer and Schmitt 1990). For example, the word "euthanasia" may be interpreted differently and confused with other end-of-life decisions and a range of situations (Marcoux, Mishara, and Durand 2007).

The second risk is related to differences in the interpretation of items by respondents and researchers. Researchers often assume that respondents make the same generalization from the item to some latent construct which the researcher had in mind. If this is not the case, then the single-item measurement can omit certain parts of the construct, which in turn would cause a risk of content under-representation (Messick 1998). For example, responses to general justifiability of the homosexuality item may involve associations with homosexual men rather than women, resulting in capturing only part of the attitude's content. Moreover, if respondents are similar in their (mis)interpretation of an item, it can cause a systematic bias, an under- or overestimation of the justifiability attitudes. This implies a bias in means, standard deviations, and magnitudes of correlations with external variables.

The multi-item instruments are more resistant to these two problems. Accordingly, we expected that the MAS items as compared to the multi-item measurement would contain a large amount of noise (H1), which would be evidence of low concurrent validity. To detect a "large" amount we used a cutoff of 50 percent of the true variance. Moreover, more general statements as compared with detailed questionnaires can activate negative stereotypes which can lead to more negative responses, or to an underestimation of the true justifiability $(\mathrm{H} 2)$. We did not have any expectations of a systematic content bias; instead we explored it in a post-hoc manner.

\section{Battery format of question}

Despite the compact form of item presentation, single-item measurement combined with the battery format of the question can undermine reliability of response (Revilla, Toninelli, and Ochoa 2017; Couper, Traugott, and Lamias 2001; Toepel and van Soest 2009). It may reduce the cognitive effort made by those responding (De Leeuw, Hox, and Dillman 2012) and increase the difficulty of answering for respondents who find it difficult to keep the instructions in mind, and pay less attention to the differences in specific items (Callegaro, Shand-Lubbers, and Dennis 2009), increasingly so with larger lists of items (Couper et al. 2013). As a result, response motivation among tired, inattentive, and less interested individuals may decrease. When such respondents face difficulties in responding, they tend to seek ways to simplify the process of participation in the survey, giving only vaguely acceptable answers, that is, using "satisficing" strategies (Simon 1956; Krosnick 1991). This may cause many undesirable behaviors, such as 
failure to differentiate between items, that is, selecting the same response options regardless of the respondent's real attitudes or item wordings (Siminski 2008); acquiescence, or agreeing with any statement; a "primacy" or "recency effect", which in the context of batteries implies choosing the first satisfactory response option and copying it to the other items (Atkinson and Shiffrin 1968); overuse of "I don't know" response category; answering randomly; skipping items; or even terminating a survey (Barge and Gehlbach 2012). Moreover, items in a battery can be perceived as conceptually related (the "near means related" heuristic), which creates a content bias (Tourangeau, Couper, and Conrad 2004). Thus, the results obtained with such instruments may reflect certain patterns of the responding behavior (i.e., their response sets, see Cronbach 1950) instead of pure respondents' attitudes. As a result, the correlation and regression coefficients as well as the conclusions based on them can be biased.

In regard to MAS, all these problems may apply. For example, the homosexuality item is eighth in the list and follows the bribery item. This may imply that, separately from the attitudes towards homosexuality, the response may also be influenced by the bribery attitude. ${ }^{1}$ Moreover, a large part of responses to this specific item may likely be a part of a generalized justifiability, that is, a general tendency to justify anything. Both of these risks invoke both random noise in data and systematic bias, undermining reliability and validity respectively. Therefore, we expected that satisficing, measured by a level of non-differentiation, shorter survey-completing time, and a general justifiability (or response set), reduces the concurrent validity of the four MAS items (H3). Here, by concurrent validity we mean the extent of the item's link with the multi-item scale. In addition, it is reasonable to expect that both the effects of satisficing and of single-item measurement reduce or bias the relations of the MAS items with external variables, such as age, religiosity, and gender (H4).

\section{METHODS}

\section{Data}

We conducted a self-administered online survey of 493 users of a Russian social network. To obtain justified conclusions, it was necessary to provide sufficient variance in moral attitudes. We used sample quotas based on age, gender, and level of religiosity as the moral attitudes demonstrated dependency on these variables (e.g. Herek 1988; Domino and Miller 1992). Age quotas were proportional to the structure of adult network users (WCIOM 2018), while in regard to gender and religiosity, only approximate correspondence to the population parameters was

\footnotetext{
${ }^{1}$ Although it is an extreme example, the overlap with the bribery item may impact the homosexuality item with a large amount of noise. Partly supporting this claim are the correlations between items that were always positive and reached up to 0.4 in some countries (EVS wave 4, own calculations).
} 
ensured. The sample was limited to active users of a social network (those who logged into their accounts every or almost every day) due to time constraints. The data were collected in April 2018. The data and the $\mathrm{R}$ code that produced all the analyses are available at https://osf.io/h5ewd/?view_only=7f1c15ce4bc54b8ebc0c5ab055b8d919

\section{Measures}

The online questionnaire included the EVS, the Wave 4 version of MAS (see Table 1) and validated multi-item measures of the analyzed concepts. In order to keep the questionnaire short enough to hold respondents' attention, we limited the number of constructs to four: suicide, prostitution, homosexuality, and euthanasia. The MAS was presented before the four multi-item scales; the euthanasia attitudes scale was placed after the suicide scale as the latter included a question regarding euthanasia. Returning to previous questions was not allowed.

The criterion instruments were selected based on the popularity of use in published studies. The selection was made using a systematic search for studies focused on scale development and demonstrable reliability and validity. Then we chose the instruments that gave similar interpretations of the constructs to the popular interpretations of the corresponding MAS items. Preference was given to shorter scales. ${ }^{2}$

To assess attitudes towards suicide, we used the subscale "suicide acceptability" of the scale "Suicide Opinion Questionnaire” (Domino, Moore, Westlake, and Gibson 1982). It includes 11 items related to the justification of committing suicide in given ways by given people (for example, people with incurable diseases or soldiers at war), as well as more general attitudes (for example, whether such a thing is "normal"). The scale includes 5 response options, from "Strongly agree" to "Strongly disagree". Reliability is indicated by a high internal consistency (alpha was 0.89 - Rogers and DeShon 1992). Validity was achieved by item selection, exploratory factor analysis, and correlations with gender (Domino and Perrone 1993), level of education (Beautrais, Horwood, and Fergusson 2004; VanSickle et al. 2016), religiosity (Domino and Miller 1992), previous thoughts about committing suicide, precedents in family, and culture (Domino 2005; Domino, Shen, and Su 2000).

To measure attitudes towards prostitution, an 8-item subscale "prostitution as normativeness/deviance" of the "Attitudes Towards Prostitutes and Prostitution Scale" (Levin

\footnotetext{
${ }^{2}$ Since instruments measuring attitudes towards suicide, prostitution, and homosexuality were not available in Russian, they were translated from English. To ensure the semantic correspondence of the translation we conducted four cognitive interviews using the "think aloud" method. We encountered the use of ambiguous, non-neutral, stylistically incorrect, or inappropriate wordings, and a bias in focus from the primary semantic categories of the question. Following this, the wording of several questions was corrected. The full questionnaire in Russian is available in the online supplement.
} 
and Peled 2011) was selected. The items describe the influence of prostitution on certain social institutions, norms, and practices (for example, on the institution of marriage or the level of drug use in society). There are 5 response options from "Fully disagree" to "Fully agree". The reliability indicated by internal consistency was 0.86 , and validity was achieved by item selection, exploratory factor analysis, correlations with perception of rape and tolerance towards certain social groups (ibid.).

Attitudes towards homosexuality were assessed using a revised short version of "The Attitudes Towards Lesbians and Gay Men Scale” (Herek 1997 [1984]) with two subscales: "attitudes towards gay men" and "attitudes towards lesbians". The 10 items describe certain feelings about homosexuals and their position in society. The respondents evaluated every item using a 5-point response scale, from "Strongly disagree" to "Strongly agree". Test-retest reliability was 0.80 ; internal consistency reliability was 0.80 for the whole scale; for the subscale “attitudes towards gay men" it was 0.89 , “attitudes towards lesbians" 0.77 . Validity was achieved by item selection and exploratory factor analysis, and indicated through correlations with religiosity, commitment to traditional gender-role relationships, family values, and gender (Herek 1988).

Attitudes towards euthanasia were measured with a 12-item scale that took into account four different end-of-life scenarios (indirect, active, and passive euthanasia, and assisted suicide) and three possible medical conditions of a patient (Siegers et al. 2017). The respondents were asked to evaluate whether doctors should be allowed to conduct the procedure, using a 4-point scale from "Should definitely be allowed" to "Should definitely not be allowed". Reliability was indicated by a high fit of confirmatory factor analysis. The scale had a high measurement invariance level across Russian and German samples. It demonstrated a significant relationship with gender, age, level of religiosity, and basic values (ibid.). Due to high correlations between items in the scale, we were able to use three subscales, each focusing on the medical condition of the patient (suffering and dying, suffering but not dying, or not dying but completely dependent).

In our sample, all the scales and subscales had acceptable internal consistency, with Cronbach's Alphas above 0.7 (see Table 2). We calculated indices for each of the scales and subscales; reverse coded items were recoded in order to make higher index scores correspond to higher justification of the aspect. 
Table 2. Cronbach's Alpha reliability for scales and subscales, and correlations of indices with single items

\begin{tabular}{|c|c|c|c|c|c|c|c|}
\hline & & & & & & $\begin{array}{r}\text { Correlatio } \\
\text { the corres } \\
\text { MAS i }\end{array}$ & $\begin{array}{l}\text { s with } \\
\text { nding } \\
\text { ms }\end{array}$ \\
\hline & Alpha & $\begin{array}{l}\text { Number } \\
\text { of items }\end{array}$ & Mean & $\begin{array}{l}\text { Standard } \\
\text { deviation }\end{array}$ & $\mathbf{N}$ & $\mathbf{r}$ & $\mathbf{N}$ \\
\hline $\begin{array}{l}\text { Attitudes towards } \\
\text { homosexuality }\end{array}$ & 0.90 & 10 & 3.04 & 1.19 & 248 & $0.77 * * *$ & 359 \\
\hline $\begin{array}{l}\text { Subscale "attitudes } \\
\text { towards gay men" }\end{array}$ & 0.88 & 5 & 2.70 & 1.37 & 311 & $0.76 * * *$ & 358 \\
\hline $\begin{array}{l}\text { Subscale "attitudes } \\
\text { towards lesbians" }\end{array}$ & 0.79 & 5 & 3.40 & 1.23 & 279 & $0.67 * * *$ & 356 \\
\hline $\begin{array}{l}\text { Attitudes towards } \\
\text { prostitution }\end{array}$ & 0.79 & 8 & 2.65 & 1.04 & 236 & $0.66 * * *$ & 369 \\
\hline $\begin{array}{l}\text { Attitudes towards } \\
\text { suicide }\end{array}$ & 0.89 & 11 & 2.44 & 1.06 & 165 & $0.54 * * *$ & 322 \\
\hline $\begin{array}{l}\text { Attitudes towards } \\
\text { euthanasia }\end{array}$ & 0.96 & 12 & 2.07 & 0.82 & 200 & $0.54 * * *$ & 293 \\
\hline $\begin{array}{l}\text { Subscale "A person is } \\
\text { incurably sick, suffering } \\
\text { and dying" }\end{array}$ & 0.92 & 4 & 2.27 & 0.91 & 239 & $0.55 * * *$ & 287 \\
\hline $\begin{array}{l}\text { Subscale "A person is } \\
\text { incurably sick, } \\
\text { suffering, but not dying } \\
\text { " }\end{array}$ & 0.95 & 4 & 1.95 & 0.89 & 261 & $0.47 * * *$ & 275 \\
\hline $\begin{array}{l}\text { Subscale "A person is } \\
\text { not suffering, is not } \\
\text { dying, but is completely } \\
\text { dependent on other } \\
\text { people” }\end{array}$ & 0.95 & 4 & 1.93 & 0.92 & 268 & $0.45 * * *$ & 265 \\
\hline
\end{tabular}

To measure satisficing tendencies, we used three indicators: non-differentiation, rushing, and response sets (Barge and Gehlbach 2012) ${ }^{3}$. Non-differentiation was indicated by the index calculated as one divided by within-individual standard deviation of the MAS items responses,

\footnotetext{
${ }^{3}$ The other two indicators of satisficing suggested by Barge and Gehlbach (2012), namely "early termination" and "skipping items", were not used in the analysis, since they could only be based on substantive questions used in the same analysis. Thus, the high values of these indicators mean that respondents did not answer a large number of questions and, as a result, summary indices for some multi-item scales could not be calculated for them.
} 
so that the higher values corresponded to lower differentiation between responses. Rushing was the time a respondent spent on answering the questionnaire, divided by the number of completed items. A response set, or a generalized justifiability, was calculated as an average of all the MAS items, excluding the four that were in the focus of the study.

In order to assess relations of the items with external variables, we employed two variables measuring religiosity, including behavioral (frequency of attending religious services) and subjective (degree of religiosity) aspects. Age was measured with a question including five age groups, and gender was a binary item.

\section{RESULTS}

\section{Degree, direction, and content of bias}

The correlations between the multi-item scales indices and the MAS items are presented in the last columns of Table 2. The correlation coefficient for the attitudes towards homosexuality had a notably high value $(\mathrm{r}=0.77, \mathrm{p}<0.001)$, which indicates an acceptable level of concurrent validity of the MAS item: it captured 59\% of the total "true" variance indicated by the corresponding multi-item scale index. Correlations between the remaining pairs of variables were moderate: for attitudes towards prostitution, the correlation was 0.66 ; for attitudes towards suicide and euthanasia it was 0.54 . Therefore, the MAS items explained smaller proportions of the "true" variance: $44 \%$ of the justification of prostitution and only $29 \%$ of attitudes towards suicide and euthanasia.

To assess the direction of bias, we plotted and compared observed versus perfect regression lines relating MAS items and indices. Figure 1 shows the joint distribution of answers to MAS items ( $\mathrm{Y}$-axis) and indices (X-axis). The dotted line indicates the perfect regression line, that is, a distribution with an ideal correlation between these two variables. It represents the score respondents would have received if their responses had not been biased. The red line indicates the empirical, observed relationship established by the loess function. The position of the empirical regression line under the theoretical one means an underestimation of the justification of one or another aspect by a MAS item, the position above the theoretical line its overestimation. 

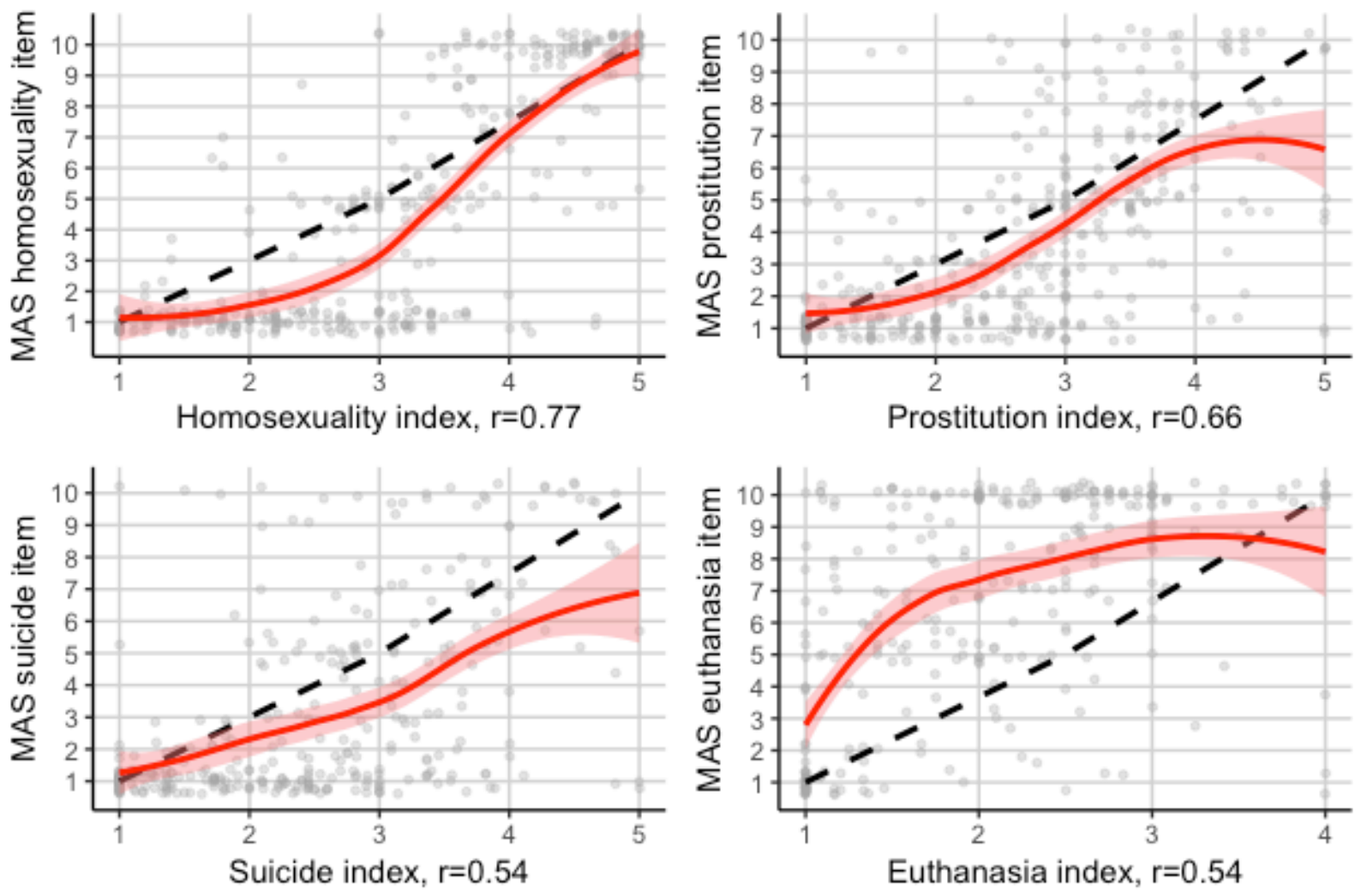

Fig. 1 The joint distribution of answers to the four MAS items and the corresponding multi-item scales indices (a dashed line is perfect regression, a solid red line is observed relationship, and the shaded area around the line is a $95 \%$ confidence interval)

Attitudes towards homosexuality. Responses to the MAS homosexuality item were fairly polarized: $69 \%$ of answers fell into the first and last categories, another $11 \%$ into the middle category (" 5 "), and only $20 \%$ were distributed among the remaining seven response categories. This caused the negative attitudes to concentrate in the lowest response option in the MAS item. The positive attitudes were, by contrast, overestimated, mostly falling in the highest category, "10". The underestimation of the lower gradations occurred more often in the sample. Therefore, in general, the MAS item systematically underestimated a positive attitude towards homosexuality.

Figure 2 plots the joint distribution of scores of the same MAS item and the two subscales measuring attitudes towards male and female homosexuality. The correlation of the general item with male homosexuality had higher value $(\mathrm{r}=0.76, \mathrm{CI}=0.71-0.81)$ than the correlation with the attitudes towards female homosexuality $(r=0.67, \mathrm{CI}=0.60-0.73)$, although the difference was not significant. However, Figure 2 demonstrates that theoretical and empirical lines for the subscale "attitudes towards gay men" are fairly close, while for the female homosexuality subscale, the empirical curve is well below the theoretical expectation. Indeed, respondents who answered strictly negatively to the single question about the justification of 
homosexuality expressed much more tolerant attitudes towards female homosexuality in the multi-item instrument.
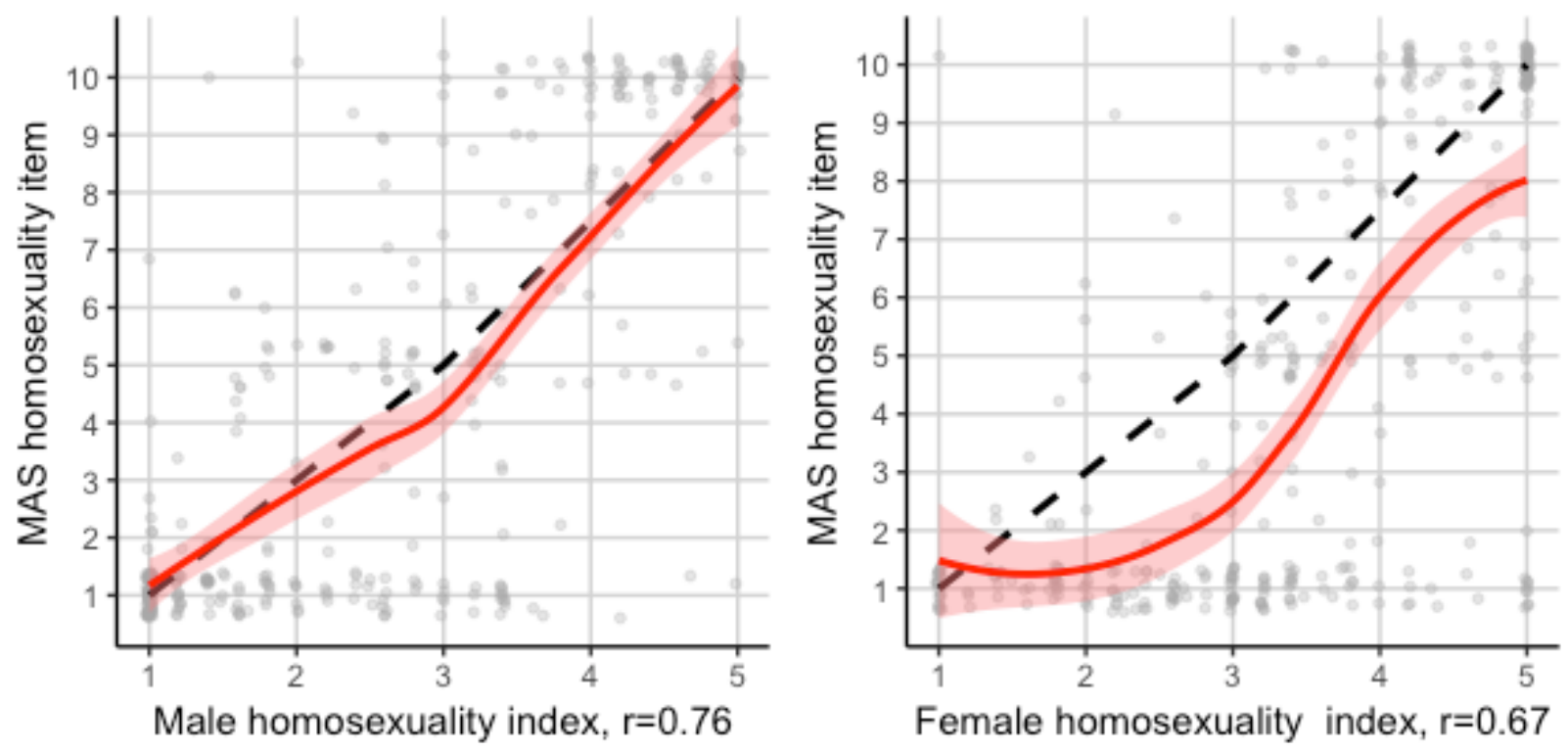

Fig. 2 The joint distribution of responses to the MAS homosexuality item and the corresponding indices of attitudes towards gays and lesbians (the dashed line is perfect regression, the solid red line is observed relationship, and the shaded area around the line is a $95 \%$ confidence interval)

Attitudes towards prostitution. The distribution of answers to the MAS item was skewed towards negative pole: $38 \%$ of respondents chose the lowest category "never justified" (" 1 "), and 10\% chose the second category. Some respondents who chose these alternatives expressed more tolerant attitudes when asked the detailed questions. As a result, the empirical line in Figure 1 is below the perfect line; thus, the MAS prostitution item systematically underestimated positive attitudes towards prostitution. At the upper part of the scale, the correlation between the MAS item and the multi-item scale index becomes even lower - after exclusion of respondents who chose the response options " 1 " and " 2 ", it dropped from 0.66 to 0.45 .

To understand the content source of such underestimation, we analyzed the individual correlations between the MAS item and the indicators from the multi-item scale (see Appendix 2 ). The strongest correlations of the general question were related to "damaging society's morals" $(\mathrm{r}=-0.57)$ and "violation of women's human dignity" $(\mathrm{r}=-0.54)$, while items that contained arguments for positive attitudes towards prostitution had the weakest correlations, for example, "stress relief" $(\mathrm{r}=0.37)$ and "preventing rape" $(\mathrm{r}=0.32)$. These results may indicate that the MAS prostitution item had fewer chances to stimulate positive argumentation, while 
being more aligned with negative arguments. If this is the case, the high values of the MAS item seem to reflect indifference, rather than truly positive attitudes.

Attitudes towards suicide. The distribution of answers to the MAS suicide item was strongly skewed towards disapproval: $54 \%$ of respondents chose the category "never justified". At the same time, more detailed questions from the multi-item scale showed less intensely negative attitudes. As a result, the MAS suicide item underestimated the positive attitudes: the empirical regression line lies below the theoretical one. As to the content of this bias (Appendix $2)$, the correlations for all statements were similar. The exception was an item associating soldiers' suicide in captivity with heroism: it was uncorrelated to responses to the MAS suicide item. Thus, we did not find content lacunae in the single-item measurement of attitudes towards suicide but recorded a weak overall correlation and the negative direction of the bias.

Attitudes towards euthanasia. The distribution of answers to the MAS euthanasia item resembled normal. Correlation coefficients with three subscales (listed in Table 2 and Figure 3) suggest that respondents perceived the MAS item as an assessment of a voluntary termination of life under the condition of strong pain and dying (corresponding $r=0.55$ ). The MAS item to a lesser extent took into account the attitudes towards euthanasia as a termination of life in the case of suffering of non-terminal disease $(r=0.47)$, or non-terminal disease that makes an individual dependent on other people $(r=0.45)$. Analysis of the correlations of the MAS item and individual statements of the multi-item scale (Appendix 2) showed no significant differences. In Figures 1 and 3, the empirical curves lie clearly above the theoretical lines. This suggests that the MAS item overestimated the justification of euthanasia compared to the more detailed questions.
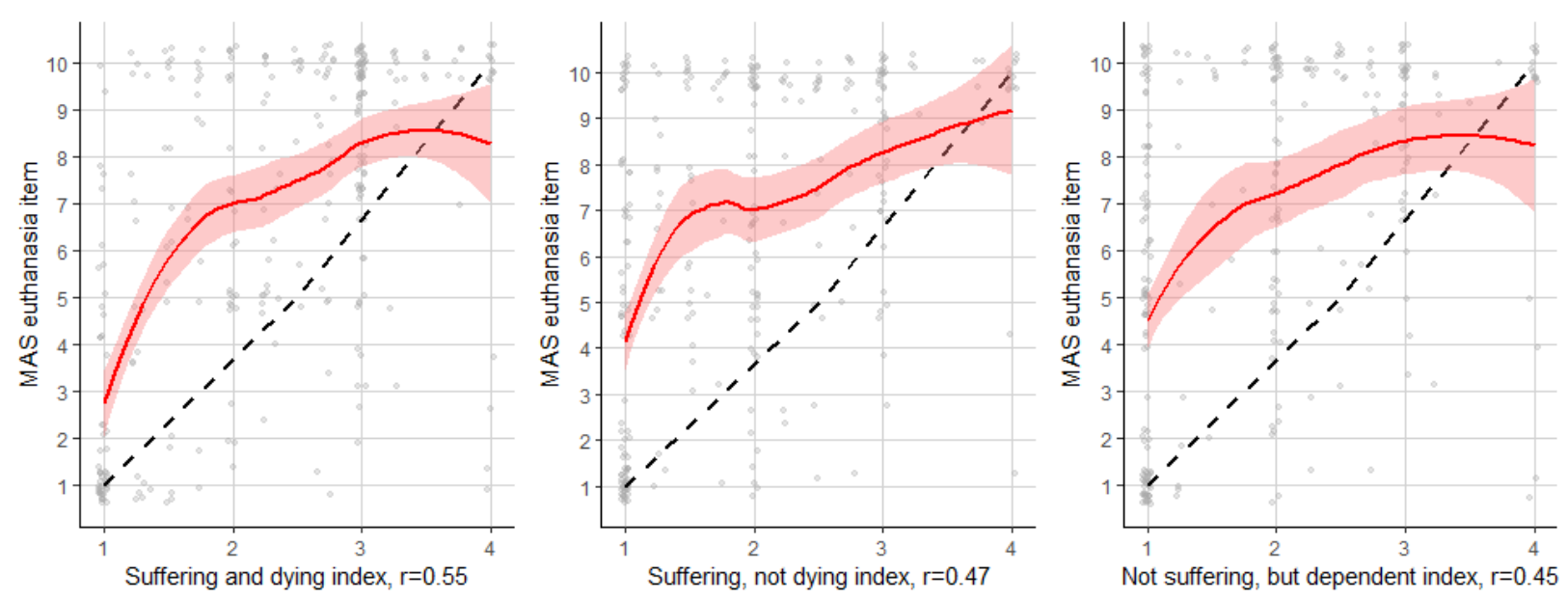

Fig. 3 The joint distribution of answers to the MAS euthanasia item and the corresponding subscale indices (the dashed line is perfect regression, the solid red line is observed relations, and the shaded area around the line is a 95\% confidence interval) 


\section{The impact of the MAS battery format}

Like the single MAS items, the questions for the selected multi-item scales followed the battery format, with Likert-type response options. To assess the isolated influences of the "true" values of the constructs provided by their multi-item measures on the MAS items, we fitted regression models for each of the analyzed MAS items, using the scores on corresponding multiitem scales and three indicators of satisficing as independent variables. The results are presented in Table 3.

Regression coefficients of multi-item indices were significant in almost all models (except for the "burden" condition of the euthanasia attitudes), showing that every MAS item represented a significant proportion of the true variance of the corresponding construct after controlling for satisficing influences. At the same time, the effects of the multi-item scales were lower than the effects of an overall justifiability for all the MAS items except homosexuality. This means that responses to the single items were not specific, mostly reflecting overall justifiability (or a response set). The overall justifiability had the greatest effect on the MAS euthanasia item: its effect was twice as large as the coefficient of the multi-item index, and this difference becomes larger for the three euthanasia subindices.

Response non-differentiation was weakly related to the higher justification of suicide, but to the lower justification of euthanasia and homosexuality (although the latter was the case only in the model with attitudes towards lesbians among independent variables). This suggests that positive responses to the MAS suicide item and negative responses to the euthanasia item were made by respondents who used a satisficing strategy. It matches the abovementioned results. Indeed, the deviations of the observed responses to the suicide items were located in the higher response categories, and for euthanasia in the lower response categories. And in line with the regression to mean statistical law, the random-driven responses tend to be closer to the middle of the scale, as compared to the true scores. Thus, non-differentiation of responses was at least partly responsible for the bias described above.

The interactions between non-differentiation and multi-item scales indices were significant and negative for homosexuality, prostitution, and suicide attitudes. This indicates that the higher non-differentiation was related to the weaker link of MAS item responses to the "true" scores represented by multi-item scales. However, this is not the case for euthanasia attitudes, where the main effects, rather than interactions of non-differentiation, were significant (and negative). Response time was insignificant in all models, both as a main effect and in the interactions with multi-item scales. 
Table 3. Standardized regression coefficients predicting battery items with indicators of overall justifiability, response differentiation, response time and multi-item scales' scores

\begin{tabular}{|c|c|c|c|c|c|c|c|c|c|}
\hline \multirow[b]{3}{*}{$\begin{array}{l}\text { Overall justifiability (MAS } \\
\text { battery mean) }\end{array}$} & \multicolumn{9}{|c|}{ Dependent variables - MAS items } \\
\hline & \multicolumn{3}{|c|}{ homosexuality } & \multirow{2}{*}{$\begin{array}{c}\text { prostitution } \\
0.64 * * * \\
(0.05)\end{array}$} & \multirow{2}{*}{$\begin{array}{c}\text { suicide } \\
0.63 * * * \\
(0.05)\end{array}$} & \multicolumn{4}{|c|}{ euthanasia } \\
\hline & $\begin{array}{c}0.29 * * * \\
(0.04)\end{array}$ & $\begin{array}{c}0.34 * * * \\
(0.04)\end{array}$ & $\begin{array}{c}0.35 * * * \\
(0.05)\end{array}$ & & & $\begin{array}{c}0.56^{* * *} \\
(0.04)\end{array}$ & $\begin{array}{c}0.56 * * * \\
(0.04)\end{array}$ & $\begin{array}{c}0.60 * * * \\
(0.04)\end{array}$ & $\begin{array}{c}0.62 * * * \\
(0.04)\end{array}$ \\
\hline $\begin{array}{l}\text { Response non-differentiation } \\
\text { (1/MAS battery sd) }\end{array}$ & $\begin{array}{l}-0.08 \\
(0.04)\end{array}$ & $\begin{array}{l}-0.04 \\
(0.04)\end{array}$ & $\begin{array}{l}-0.14^{*} \\
(0.06)\end{array}$ & $\begin{array}{c}0.08 \\
(0.04)\end{array}$ & $\begin{array}{l}0.12^{*} \\
(0.05)\end{array}$ & $\begin{array}{c}-0.21 * * * \\
(0.04)\end{array}$ & $\begin{array}{c}-0.22 * * * \\
(0.04)\end{array}$ & $\begin{array}{c}-0.22 * * * \\
(0.04)\end{array}$ & $\begin{array}{c}-0.26^{* * * *} \\
(0.05)\end{array}$ \\
\hline Response time & $\begin{array}{l}-0.02 \\
(0.03)\end{array}$ & $\begin{array}{l}-0.03 \\
(0.03)\end{array}$ & $\begin{array}{l}-0.01 \\
(0.04)\end{array}$ & $\begin{array}{c}0.03 \\
(0.04)\end{array}$ & $\begin{array}{c}0.04 \\
(0.05)\end{array}$ & $\begin{array}{l}-0.04 \\
(0.04)\end{array}$ & $\begin{array}{l}-0.05 \\
(0.04)\end{array}$ & $\begin{array}{l}-0.04 \\
(0.04)\end{array}$ & $\begin{array}{l}-0.03 \\
(0.04)\end{array}$ \\
\hline $\begin{array}{l}\text { Attitudes to homosexuality, } \\
\text { index }\end{array}$ & $\begin{array}{c}0.60 * * * \\
(0.04)\end{array}$ & & & & & & & & \\
\hline$\times$ non-differentiation & $\begin{array}{c}-0.19 * * * \\
(0.04)\end{array}$ & & & & & & & & \\
\hline$\times$ response time & $\begin{array}{l}-0.03 \\
(0.02)\end{array}$ & & & & & & & & \\
\hline
\end{tabular}

Attitudes to male

homosexuality, subindex

$$
\begin{gathered}
0.60 * * * \\
(0.03) \\
-0.15 * * * \\
(0.03) \\
-0.02 \\
(0.02)
\end{gathered}
$$$$
\times \text { non-differentiation }
$$

Attitudes to female homosexuality, subindex

$$
\begin{gathered}
0.45^{* * *} \\
(0.04) \\
-0.20 * * * \\
(0.04) \\
-0.01 \\
(0.03)
\end{gathered}
$$

Attitudes to prostitution, index

$$
\begin{gathered}
0.25^{* * *} \\
(0.04) \\
-0.09 * * \\
(0.03) \\
-0.01 \\
(0.03)
\end{gathered}
$$

Attitudes to suicide, index

$$
\begin{gathered}
0.25 * * * \\
(0.05) \\
-0.09 * * \\
(0.04) \\
-0.01 \\
(0.05)
\end{gathered}
$$

Attitudes to euthanasia, index

$$
\begin{gathered}
0.18 * * * \\
(0.04) \\
-0.03 \\
(0.03)
\end{gathered}
$$




\begin{tabular}{cccc}
\hline & \multicolumn{2}{c}{ Dependent variables - MAS items } \\
& homosexuality & prostitution suicide & euthanasia \\
\hline$\times$ response time & & -0.04 & $(0.04)$
\end{tabular}

Attitudes to euthanasia:

subscale "A person is incurably sick, suffering and dying"

$$
\begin{aligned}
& \times \text { non-differentiation } \\
& \times \text { response time }
\end{aligned}
$$

Attitudes to euthanasia: subscale "A person is incurably sick, suffering, but not dying"

$$
\begin{aligned}
& \times \text { non-differentiation } \\
& \times \text { response time }
\end{aligned}
$$

Attitudes to euthanasia: subscale "A person is not suffering, is not dying, but is completely dependent on other people"

$$
\begin{aligned}
& \times \text { non-differentiation } \\
& \times \text { response time }
\end{aligned}
$$

\begin{tabular}{lccccccccc} 
Constant & -0.02 & 0.01 & -0.04 & -0.04 & -0.02 & -0.01 & -0.02 & -0.04 & -0.05 \\
& $(0.03)$ & $(0.03)$ & $(0.04)$ & $(0.03)$ & $(0.04)$ & $(0.04)$ & $(0.04)$ & $(0.04)$ & $(0.04)$ \\
\hline Observations & & & & & & & & & \\
Adjusted R2 & 359 & 358 & 356 & 369 & 322 & 293 & 287 & 275 & 265 \\
& 0.69 & 0.71 & 0.56 & 0.61 & 0.53 & 0.64 & 0.67 & 0.64 & 0.64
\end{tabular}

Note: $* p<0.05 ; * * p<0.01 ; * * * p<0.001$

\section{The MAS items' bias of correlations with external variables}

To assess the criterion validity of the MAS items as compared to the multi-item scales, we computed correlations with the subjective level of religiosity, frequency of religious services attendance, age, and gender of respondents. Table 4 presents these correlations: the first column lists correlations with MAS items, the second with multi-item indices of the corresponding scales, and the third the difference between these correlations. The correlations of MAS items and multi-item indices with external variables appeared to be very similar. Only the correlations 
of religious attendance with attitudes towards euthanasia were significantly different across the two measures: when using the MAS item, the correlation was -0.54 , while for the multi-item scale it was -0.32 , resulting in the statistically significant difference of -0.22 .

Table 4. Correlation coefficients between multi-item scales, MAS items, and external variables

\begin{tabular}{|c|c|c|c|}
\hline & $\begin{array}{c}\text { Correlation with single MAS } \\
\text { item }\end{array}$ & $\begin{array}{l}\text { Correlation with multi-item } \\
\text { index }\end{array}$ & Difference \\
\hline \multicolumn{4}{|c|}{ Subjective religiosity } \\
\hline Homosexuality & $-0.28 * * *$ & $-0.37 * * *$ & 0.08 \\
\hline Prostitution & $-0.33 * * *$ & $-0.37 * * *$ & 0.04 \\
\hline Suicide & $-0.35 * * *$ & $-0.45 * * *$ & 0.10 \\
\hline Euthanasia & $-0.48 * * *$ & $-0.40 * * *$ & -0.08 \\
\hline \multicolumn{4}{|c|}{ Frequency of church attendance } \\
\hline Homosexuality & $-0.29 * * *$ & $-0.36 * * *$ & 0.07 \\
\hline Prostitution & $-0.31 * * *$ & $-0.33 * * *$ & 0.02 \\
\hline Suicide & $-0.32 * * *$ & $-0.40 * * *$ & 0.08 \\
\hline Euthanasia & $-0.54 * * *$ & $-0.32 * * *$ & $-0.22 *$ \\
\hline \multicolumn{4}{|l|}{ Age } \\
\hline Homosexuality & $-0.11 *$ & -0.07 & -0.04 \\
\hline Prostitution & -0.08 & 0.00 & -0.08 \\
\hline Suicide & $-0.17 * * *$ & $-0.17 * *$ & 0.00 \\
\hline Euthanasia & $-0.13^{*}$ & $-0.13^{*}$ & 0.00 \\
\hline \multicolumn{4}{|l|}{ Gender (female) } \\
\hline Homosexuality & 0.09 & 0.01 & 0.07 \\
\hline Prostitution & $-0.27 * * *$ & $-0.19 * * *$ & -0.08 \\
\hline Suicide & $-0.11^{*}$ & $-0.14 * *$ & 0.03 \\
\hline Euthanasia & $-0.16 * *$ & $-0.11 *$ & -0.05 \\
\hline
\end{tabular}

Note: $* p<0.05 ; * * p<0.01 ; * * * p<0.001$ 


\section{DISCUSSION AND CONCLUSION}

In this study, we aimed to assess concurrent and content validity of the MAS measures of the justifiability of homosexuality, prostitution, suicide, and euthanasia. To identify potential bias, we compared the responses to the four MAS items with the results of the multi-item measurement instruments with known validity and reliability, examining the degree, direction, and content of deviations, as well as their correlations to the indicators of satisficing and sociodemographic variables. Analysis of the Russian respondents demonstrated that the magnitude and the direction of these biases differed substantially across items.

In line with hypothesis H1, three MAS items demonstrated low concurrent validity, covering less than 50 percent of the true variance. At the same time, the single-item measurement of attitudes towards homosexuality showed an acceptable level of concurrent validity. Content-wise, the MAS homosexuality item was somewhat more closely related to the attitudes towards male rather than female homosexuality. Such bias might be caused by the fact that in European countries (including Russia), gay men have more media coverage than lesbians (cf. Council of Europe 2011). This may have affected the perception of the general homosexuality item as a predominantly male feature. This conclusion is worth testing in future research. Overall, the MAS item proved to be a good indicator of justifiability of male homosexuality, and not as good an indicator of attitudes toward women's homosexuality.

In line with hypothesis H2, MAS underestimated the positive attitudes towards homosexuality, prostitution, and suicide. We suggest that this happened because each of the four MAS items under-represents a content of the corresponding constructs. The MAS homosexuality item underestimated positive attitudes because, as mentioned above, it was biased towards male homosexuality, which, on average, was judged more negatively than female homosexuality. The MAS prostitution item had stronger associations with negative argumentation, such as harm to society's morals and violation of women's dignity, while positive arguments had less apparent impact on responses. As a result, the MAS item underestimated the justifiability of prostitution. Similarly, the assessment of the justification of suicide might be negatively biased due to absence of positive argumentation.

This interpretation is in line with the stereotype activation theory (Bargh, Chen, and Burrows 1996), which asserts that priming with stereotype-related attributes leads to automatic stereotype-related reactions. In our case, the respondents were presented with very general and undetailed concepts (e.g. "prostitution"), which were likely to invoke negative stereotypes and therefore led to the overly negative responses. In contrast, when the same respondents were presented with the detailed questions concerning different aspects of the concepts, their responses were less automatic, less affected by stereotypes, and, likely, more thoughtful. 
Unlike the other MAS items, the euthanasia item overestimated justifiability. One explanation comes from the incomplete content validity of the item: it taps more into euthanasia of suffering, incurably sick and dying people, while attitudes towards life termination in more complex situations were not covered. However, even an attitude to the situation of a sick and dying person's life termination was overestimated by the MAS item. Moreover, it contradicts the findings of Magelssen et al. (2016), who showed that context-focused questions (such as the multi-item scale in our case) tended to produce more favorable responses as compared with concept-based ones (such as the MAS item). This discrepancy could be caused by the cultural differences between participants (Magelssen et al. focused on a Norwegian sample, while we studied Russians). But a more likely explanation is the influence of satisficing: responses to the euthanasia MAS item had the lowest concurrent validity and appeared to be highly affected by the overall justifiability as well as the response non-differentiation. This implies that the respondents put less cognitive effort in answering this item and the resulting scores contained a large portion of the overall justifiability. If euthanasia was truly less justified than the other MAS items, the overall justifiability and non-differentiation could lead to an overestimation of this attitude.

In line with hypothesis $\mathrm{H} 3$, we found strong effects of satisficing tendencies related to the "battery" format of MAS: temporal and visual proximity of the items in the survey brought a homogeneous structure of responses. The impact of overall justifiability and non-differentiation was less pronounced for the homosexuality MAS item, while it drastically impacted the other three items. Prostitution, suicide, and especially euthanasia MAS items were predicted by general justifiability (i.e. overall mean on the rest of the 16 MAS items) even better than by the multi-item scales measuring corresponding constructs. Interactions between multi-item scales and a non-differentiation index were significant for homosexuality, prostitution, and suicide MAS items, indicating that non-differentiation is a substantial problem that deteriorates items' validity.

In contrast with hypothesis $\mathrm{H} 4$, the correlations with external variables did not detect significant differences between MAS items and multi-item scales. This suggests an optimistic conclusion that the predictive power of the MAS items is fair. However, this part of our study was limited to four predictors and the conclusion may change if we include another set of external variables.

In general, our findings showed that the four MAS items have a number of characteristics that can potentially lead to the deterioration of the adequacy of the conclusions, interpretations, and actions based on these scores. Summarizing the issues, we would highlight two most important problems with the use of single MAS items. The first problem is partial content 
validity, which implies that MAS items tackle only a small proportion of the true variance of attitudes, and this variance is bound to stereotype-specific content of the construct. It can lead to over- or underestimation of the mean justifiability. Second, MAS items may lack specificity, that is, they may be better indicators of general justifiability rather than attitudes towards specific aspects. Thus, the data obtained with the single MAS items can only provide a very rough, approximate, and not very domain-specific assessment of the respondents' attitudes.

As mentioned above, single MAS items were often used in a way that ignores their potential limitations. Conclusions based on such measurement of general attitudes towards one or another aspect of life may be less justified. However, our findings by no means imply that the data collected with MAS are useless. Moreover, we showed that correlations with some external variables were not affected by the format of the items. It is possible that the degree of bias is negligible when used to measure more abstract constructs, such as "general justifiability", or even more specific concepts, such as "tolerance towards sexual and reproductive issues", as measured by several items from this battery. ${ }^{4}$

This study has several limitations. First, due to resource constraints, the analysis involved only four of the twenty MAS items. Thus, the generalization of the conclusions to the other items in the battery may be questionable. Second, for the same reason, a sample from only one country was used. Therefore, we cannot be sure that the detected bias of MAS items has the same values in different countries. Nevertheless, it is clear that between-country differences in the interpretation of concepts and translation into different languages increase measurement error, and therefore the results of the present work could underestimate (rather than overestimate) the extent of bias in the measurement of these four constructs. In addition, we used an online survey with a self-administered questionnaire, whereas the reference studies, EVS and WVS, used faceto-face interviews to collect data. Potential differences in respondent behavior caused by different modes of data collection could lead to imprecise estimation of the studies' validity. The fact that both MAS and criterion scales were conducted using the same data collection mode partly compensates for this limitation. As mentioned above, the selected multi-item scales could be affected by the battery format. But, firstly, they had demonstrated reliability and validity, and, secondly, some of them used reverse-coded items, which reduce the level of satisficing in questions for these scales. Another limitation may be related to the specifics of the multi-item scales chosen as criterion variables, which may be imperfect. However, they can clearly be considered more valid and reliable than single MAS items.

\footnotetext{
${ }^{4}$ This assumption is based on the strength and the stability of the intercorrelations of all the MAS items. Still, care must be taken with interpretations, because a general justifiability score may be too high (as some items overestimated the real level of justifiability), or reflect a response set, rather than attitudes.
} 
In general, we would recommend that researchers use MAS items with caution. When interpreting the results based on single items, it is reasonable to take into account the content biases they were found to have. Likewise, when deciding on the measurement model, ideally, one should avoid using single-item measures in favor of more general concepts. This cannot guarantee the prevention of biased conclusions, but may help avoid them. 


\section{REFERENCES}

Adamczyk, A., Pitt, C.: Shaping attitudes about homosexuality: The role of religion and cultural context. Soc. Sci. Res., 38 (2), 338-351 (2009)

Alm, J., Torgler, B.: Culture differences and tax morale in the United States and in Europe. J. of Econom. Psychol., 27 (2), 224-246 (2006)

Alwin, D.F., Krosnick, J.A.: The reliability of survey attitude measurement: the influence of question and respondent attributes. Sociol. Methods \& Res. 20 (1), 139-181 (1991)

Atkinson, R.C., Shiffrin, R.M.: Human memory: a proposed system and its control processes. In: Psychology of learning and motivation (vol. 2), pp. 89-195. Academic Press (1968)

Barge, S., Gehlbach, H.: Using the theory of satisficing to evaluate the quality of survey data. Res. in High. Educ., 53 (2), 182-200 (2012)

Bargh, J. A., Chen, M., Burrows, L.: Automaticity of social behavior: Direct effects of trait construct and stereotype activation on action. J. of Pers. and Soc. Psychol., 71(2), 230 (1996)

Beautrais, A.L., John Horwood, L., Fergusson, D. M.: Knowledge and attitudes about suicide in 25-year-olds. Aust. and N. Z. J. of Psychiatry, 38 (4), 260-265 (2004)

Callegaro, M., Shand-Lubbers, J., Dennis, J.M.: Presentation of a single item versus a grid: effects on the vitality and mental health scales of the SF-36v2 health survey. 64th Annual Conference of the American Association for Public Opinion Research (AAPOR) (2009). http://www.asasrms.org/Proceedings/y2009/Files/400045.pdf Accessed 24 March 2019

Cao, L., Lu, R., Mei, X.: Acceptance of prostitution and its social determinants in Canada. Int. J. of Offender Ther. and Comp. Criminol., 61 (10), 1171-1190 (2017)

Cao, L., Zhao, R.: The impact of culture on acceptance of soft drugs across Europe. J. of Crim. Justice, 40 (4), 296-305 (2012)

Chen, C.: Are workers more likely to be deviant than managers? A cross-national analysis. J. of Bus. Ethics, 123 (2), 221-233 (2014)

Churchill, Jr, G.A.: A paradigm for developing better measures of marketing constructs. J. of Mark. Res., 16 (1), 64-73 (1979)

Council of Europe: Discrimination based on sexual orientation and gender identity in Europe. IOP Publishing COE https://rm.coe.int/discrimination-on-grounds-of-sexual-orientationand-gender-identity-in/16809079e2 Accessed 26 May 2018

Couper, M.P. et al.: The design of grids in web surveys. Soc. Sci. Comput. Rev., 31 (3), $322-345(2013)$ 
Couper, M.P., Traugott, M.W., Lamias, M. J.: Web survey design and administration. Public Opin. Q., 65 (2), 230-253 (2001)

Crissman, P.: Temporal change and sexual difference in moral judgments. J. of Soc. Psychol., 16 (1), 29-38 (1942)

Cronbach, L.J.: Further evidence on response sets and test design. Educ. and Psychol. Meas., 10 (1), 3-31 (1950)

De Leeuw, E.D., Hox, J., Dillman, D.: International handbook of survey methodology. Routledge (2012)

Domino, G., Miller, K.: Religiosity and attitudes towards suicide. OMEGA-J. of Death and Dying, 25 (4), 271-282 (1992)

Domino, G., Moore, D., Westlake, L., Gibson, L.: Attitudes towards suicide: a factor analytic approach. J. of Clin. Psychol., 38 (2), 257-262 (1982)

Domino, G., Perrone. L.: Attitudes towards suicide: Italian and United States physicians. OMEGA-J. of Death and Dying, 27 (3), 195-206 (1993)

Domino, G., Shen, D., Su, S.: Acceptability of suicide: attitudes in Taiwan and in the United States. OMEGA-J. of Death and Dying, 40 (2), 293-306 (2000)

Domino, G.: Cross-cultural attitudes towards suicide: the SOQ and a personal odyssey. Arch. of Suicide Res., 9 (2), 107-122 (2005)

EVS: About EVS. IOP Publishing European Values Study https://europeanvaluesstudy.eu/about-evs/ Accessed 11 April 2018

Fuchs, C., Diamantopoulos, A.: Using single-item measures for construct measurement in management research: conceptual issues and application guidelines. Die Betriebswirtschaft, 69 (2), 195-210 (2009)

Gorsuch, R.L., McPherson, S.E.: Intrinsic/extrinsic measurement: I/E-revised and singleitem scales. J. for the Sci. Study of Relig., 28 (3), 348-354 (1989)

Harding, S., Phillips, D., Fogarty, M.P.: Contrasting values in Western Europe: Unity, diversity and change. Macmillan Pub. Co., London (1986)

Herek, G.M. Heterosexuals' attitudes towards lesbians and gay men: correlates and gender differences. J. of Sex Res., 25 (4), 451-477 (1988)

Herek, G.M.: The attitudes towards lesbians and gay men (ATLG) scale. In Davis, C.M., Yarber, W.H., Bauserman, R., Schreer, G., Davis S.L. (eds.) Sexuality-related measures: A compendium, pp. 392-394. Sage Publications, Thousand Oaks, CA (1997)

Inglehart, R., Baker, W.E.: Modernization, cultural change, and the persistence of traditional values. Am. Sociol. Rev., 65 (1), 19-51 (2000) 
Kalmijn, M.: Country differences in the effects of divorce on well-being: the role of norms, support, and selectivity. Eur. Soc. Rev., 26 (4), 475-490 (2009)

Krosnick, J.A.: Response strategies for coping with the cognitive demands of attitude measures in surveys. Appl. Cogn. Psychol., 5 (3), 213-236 (1991)

Levin, L., Peled, E.: The attitudes towards prostitutes and prostitution scale: a new tool for measuring public attitudes towards prostitutes and prostitution. Res. on Soc. Work Pract., 21 (5), $582-593(2011)$

Lottes, I.L., Alkula, T.: An investigation of sexuality-related attitudinal patterns and characteristics related to those patterns for 32 European countries. Sex. Res. and Soc. Policy, 8 (2), 77-92 (2011)

Magelssen, M., Supphellen, M., Nortvedt, P., Materstvedt, L.J.: Attitudes towards assisted dying are influenced by question wording and order: a survey experiment. BMC Med. Ethics, 17 (1), (2016)

Marcoux, I., Mishara, B.L., Durand, C.: Confusion between euthanasia and other end-oflife decisions: influences on public opinion poll results. Can. J. of Public Health, 235-239 (2007)

Mavletova, A., Couper, M.P., Lebedev, D.: Grid and Item-by-Item Formats in PC and Mobile Web Surveys. Soc. Sci. Comput. Rev., 36 (6), 647-668 (2018)

Messick, S.: Consequences of test interpretation and use: the fusion of validity and values in psychological assessment. ETS Research Report Series, 3-20 (1998)

Revilla, M., Toninelli, D., Ochoa, C.: An experiment comparing grids and item-by-item formats in web surveys completed through PCs and smartphones. Telemat. and Inform., 34 (1), 30-42 (2017)

Rogers, J.R., DeShon, R.P.: Reliability of the suicide opinion questionnaire. Paper presented at the Silver Anniversary Conference of the American Association of Suicidology, 1-7 (1992)

Rossiter, J.R.: The C-OAR-SE procedure for scale development in marketing. Int. J. of Res. in Mark., 19 (4), 305-335 (2002)

Rudnev, M., Savelkaeva, A.: Public support for the right to euthanasia: Impact of traditional religiosity and autonomy values across 37 nations. Int. J. of Comp. Sociol., 59(4), 301318 (2018)

Saris, W.E., Gallhofer, I.N.: Design, evaluation, and analysis of questionnaires for survey research. Second Edition. John Wiley \& Sons, Hoboken, NJ (2014)

Siegers, P., Rudnev, M., Beckers, T., Henseler, A.-K. A new instrument for measuring normative views on euthanasia: structure, model and measurement invariance in Russia and 
Germany. Presentation at the XVIII April international academic conference on economic and social development (2017)

Siminski, P.: Order effects in batteries of questions. Qual. and Quant., 42 (4), 477-490 (2008)

Simon, H.A.: Rational choice and the structure of the environment. Psychol. Rev., 63 (2), 129-138 (1956)

Stack, S., Kposowa, A.J.: Culture and Suicide Acceptability: A Cross-National, Multilevel Analysis. The Sociol. Q., 57 (2), 282-303 (2016)

Steyer, R., Schmitt, M.J.: Latent state-trait models in attitude research. Qual. and Quant., 24 (4), 427-445 (1990)

Štulhofer, A., Rimac, I.: Determinants of homonegativity in Europe. J. of Sex Res., 46 (1), 24-32 (2009)

Toepoel, V., Das, M., Soest, A.V.: Design of web questionnaires: the effects of the number of items per screen. Field Methods, 21 (2), 200-213 (2009)

Tourangeau, R., Couper, M.P., Conrad, F.: Spacing, position, and order: interpretive heuristics for visual features of survey questions. Public Opin. Q., 68 (3), 368-393 (2004)

VanSickle, M. et al.: Principal component analysis of the suicide opinion questionnaire in a US military sample of Marine Corps non-commissioned officers. Mil. Medicine, 181 (7), 672679 (2016)

Vauclair, C.-M., Fischer, R.: Do cultural values predict individuals' moral attitudes? A cross-cultural multilevel approach Eur. J. of Soc. Psychol., 41 (5), 645-657 (2011)

WCIOM: Press release No.3577. For each age - its network. IOP Publishing WCIOM. https://wciom.ru/index.php?id=236\&uid=116691 Accessed 17 April 2018

Welzel, C. Freedom rising. Cambridge University Press, London (2013)

WVS: Welcome to the World Values Survey site. IOP Publishing World Values Survey http://www.worldvaluessurvey.org/wvs.jsp Accessed 08 April 2018 


\section{Appendix 1}

Distribution of respondents by sex in age groups

\begin{tabular}{ccccc}
\hline Age group & $\begin{array}{c}\text { Number of } \\
\text { respondents }\end{array}$ & $\begin{array}{c}\text { Percentage of the total } \\
\text { number }\end{array}$ & \multicolumn{2}{c}{ Gender } \\
Male & Female \\
\hline 18 -24 years & 145 & $30 \%$ & $49 \%$ & $51 \%$ \\
$25-34$ years & 193 & $39 \%$ & $44 \%$ & $56 \%$ \\
$35-44$ years & 72 & $15 \%$ & $38 \%$ & $62 \%$ \\
$45-59$ years & 63 & $13 \%$ & $40 \%$ & $60 \%$ \\
More than 60 years & 17 & $3 \%$ & $53 \%$ & $47 \%$ \\
\hline
\end{tabular}




\section{Appendix 2}

Individual correlations between single MAS items and individual statements of the corresponding multi-item scales

$$
\text { Item wording }
$$

r

\section{Correlations with MAS homosexuality item}

I think male homosexuals are disgusting

$-0.64 * * * 349$

Male homosexuality is a perversion

Male homosexuality is a natural expression of sexuality in men

Sex between two men is just plain wrong

Male homosexuality is merely a different kind of lifestyle that should not be condemned

Lesbians just can't fit into our society

$-0.54 * * * \quad 341$

State laws against private sexual behavior between consenting adult women should be abolished

Female homosexuality is a sin

Female homosexuality in itself is no problem unless society makes it a problem

Correlations with MAS prostitution item

Prostitution is important for teaching teenage boys about sexuality

Prostitution increases drug use in society

Without prostitution more women would get raped

Prostitution damages society's morals

$-0.57 * * * \quad 332$

Prostitution is a violation of women's human dignity

$-0.54 * * * \quad 330$

Prostitution provides men with stress relief

Prostitution increases the rate of sexually transmitted diseases

Prostitution harms the institution of marriage

$-0.52 * * *$

\section{Correlations with MAS suicide item}

People with incurable diseases should be allowed to commit suicide in a dignified manner

Suicide is an acceptable means to end an incurable illness

$0.46 * * * \quad 283$

Some people are better off dead

$0.32 * * * \quad 278$

Suicide is acceptable for aged and infirm persons

We should have "suicide clinics" where people who want to die could do so in a painless and private manner

In times of war, for a captured soldier to commit suicide is an act of heroism

There may be situations where the only reasonable resolution is suicide

Suicide is normal behavior

Sometimes suicide is the only escape from life's problems

If someone wants to commit suicide, it is their business and we should not interfere 


\section{Correlations with MAS euthanasia item}

a person with an incurable and painful illness, from which he or she will die-for example, someone dying of cancer: Now think about a case in which the same person demands of the doctors to receive a high dose of medication that could indirectly lead to a premature death

a person with an incurable and painful illness, from which he or she will die-for example, someone dying of cancer: Do you think that a doctor should, in this case, be allowed to stop life-extending measures, such as artificial respiration, nourishment, or drug delivery, if the person asks for it?

a person with an incurable and painful illness, from which he or she will die-for example, someone dying of cancer: Again, think about the same person: Should a doctor be allowed to end a person's life by giving him or her a deadly amount of drugs if the person asks for it? a person with an incurable and painful illness, from which he or she will die-for example, someone dying of cancer: Now please think about a case in which the same person wants to receive a deadly amount of drugs from the doctor so that the person can take the drugs by him or herself to end his or her life. Should a doctor be allowed to do so, if the patient wishes for it?

a person with an incurable and painful illness from which he or she will not die: Now think about a case in which the same person demands of the doctors to receive a high dose of medication that could indirectly lead to a premature death

a person with an incurable and painful illness from which he or she will not die Do you think that a doctor should, in this case, be allowed to stop life-extending measures, such as artificial respiration, nourishment, or drug delivery, if the person asks for it?

a person with an incurable and painful illness from which he or she will not die: Again, think about the same person: Should a doctor be allowed to end a person's life by giving him or her a deadly amount of drugs if the person asks for it?

a person with an incurable and painful illness from which he or she will not die: Now please think about a case in which the same person wants to receive a deadly amount of drugs from the doctor so that the person can take the drugs by him or herself to end his or her life. Should a doctor be allowed to do so, if the patient wishes for it?

a person who neither suffers from unbearable pain nor is close to death. But this person is, regarding all basic needs, completely dependent on other people; for example, this person cannot feed or wash him or herself and cannot use the bathroom by him or herself.: Now think about a case in which the same person demands of the doctors to receive a high dose of medication that could indirectly lead to a premature death a person who neither suffers from unbearable pain nor is close to death. But this person is, regarding all basic needs, completely dependent on other people; for example, this person cannot feed or wash him or herself and cannot use the bathroom by him or herself.: Do you think that a doctor should, in this case, be allowed to stop life-extending measures, such as artificial respiration, nourishment, or drug delivery, if the person asks for it?

a person who neither suffers from unbearable pain nor is close to death. But this person is, regarding all basic needs, completely dependent on other people; for example, this person cannot feed or wash him or herself and cannot use the bathroom by him or herself:: Again, think about the same person: Should a doctor be allowed to end a person's life by giving him or her a deadly amount of drugs if the person asks for it?

a person who neither suffers from unbearable pain nor is close to death. But this person is, regarding all basic needs, completely dependent on other people; for example, this person cannot feed or wash him or herself and cannot use the bathroom by him or herself.: Now please think about a case in which the same person wants to receive a deadly amount of drugs from the doctor so that the person can take the drugs by him or herself to end his or her life. Should a doctor be allowed to do so, if the patient wishes for it? 